\title{
Da homogeneidade biológica à heterogeneidade cultural: o papel da construção de significados no desenvolvimento humano*
}

Howard A. Smith**

\section{A psicologia infantil, como a própria criança, é uma invenção cultural (William Kessen, 1983)}

O objetivo deste artigo é, em primeiro lugar, defender duas importantes teses sobre o desenvolvimento humano e, em segundo lugar, apresentar as implicações pertinentes para a prática educacional. A tese inicial é de que os seres humanos de todas as culturas possuem o mesmo potencial biológico para desenvolver suas habilidades de sobrevivência em ambientes físicos e culturais diversos. Isto não significa negar o fato de que indivíduos dentro de uma mesma cultura demonstram ampla variação em termos de aptidões específicas nem que coletividades de diferentes culturas possam ocasionalmente diferir em melhores predisposições biológicas devido a acomodações a habitats particulares, no decorrer de várias gerações. Entretanto, dados coletados nas duas últimas décadas têm revelado alguns traços biológicos importantes que dão apoio ao argumento de

* Este artigo se baseia em um trabalho apresentado no encontro da American Educational Research Association, em Nova York, EUA, em abril de 1996.

** Ph.D, Faculty of Education, Queen's University, Canadá. Trad.: Ph.D José Erasmo Gruginski, Departamento de Letras Estrangeiras Modernas, SCHLA, UFPR. 
que os princípios fundamentais do desenvolvimento humano são universalmente os mesmos (cf., Edelman, 1987).

A segunda tese deste trabalho é a de que o desenvolvimento humano nos campos emocional, volitivo e cognitivo depende de significados construídos dentro de contextos culturais heterogêneos. Defenderei a idéia de que o desenvolvimento humano é impulsionado menos pelos traços biológicos ligados à idade do que pelas experiências culturais heterogêneas que o próprio aprendiz tornou significativas. Com o suporte de pesquisas de uma variedade de fontes, esboçarei três proposições resultantes: (a) o desenvolvimento humano depende primordialmente do crescimento constante de significados que são mediados por signos (Vygotzky, 1978), (b) a construção de significados através de signos é embasada contextualmente, derivada socioculturalmente e motivada por necessidades de competência (Cole, 1990; Smith, 1992), e (c) no decorrer de toda a vida de um indivíduo, os significados são negociados através de atividades com outros membros da cultura (Lave and Wenger, 1991). Na seção final do trabalho são apresentadas várias implicações para a educação multicultural.

\section{A homogeneidade do potencial biológico para o desenvolvimento}

Avanços recentes no conhecimento de substratos neurônicos, físicos e bioquímicos da aprendizagem e desenvolvimento humanos têm surgido principalmente do trabalho de Gerald Edelman. Cientista agraciado com o Prêmio Nobel pelo seu estudo do sistema imunológico, Edelman recentemente voltou sua atenção para as mudanças no sistema nervoso humano. Em uma série de importantes publicações (e.g., Edelman, 1987, 1992) que foram convincentemente resumidas por outros (e.g., Sacks, 1993; Sylwester, 1995), foi lançado o alicerce para uma nova compreensão de como os seres humanos se desenvolvem.

Edelman (1987), por exemplo, esboçou uma teoria biológica da percepção individual que tratava do desenvolvimento multifacetado dos grupos neurônicos. Em toda sua argumentação, Edelman enfatizou a natureza altamente variável do sistema nervoso, o qual - ao invés de ser configurado a priori - é configurado de acordo com as experiências do ambiente. 
No que se refere ao indivíduo, Edelman defendeu ainda que "o cérebro é organizado dinamicamente em populações de células que contêm redes individualmente variantes, cuja estrutura e função são selecionadas por meios diferentes durante o desenvolvimento e o comportamento" (páginas 4-5). Mais tarde defendeu a existência de mecanismos epigenéticos: "embora restritos por imposições genéticas, os eventos que ocorrem nos estágios tanto desenvolvimentais como experienciais da seleção levam, com o tempo, a aumentos tanto na heterogeneidade como na diversidade espacial das células e das estruturas celulares" (p. 73). Estas afirmações dão suporte à direção das crenças de John Locke, no século XVII, que enfatizava o papel da experiência sensorial no desenvolvimento e declarava que o padrão de desenvolvimento individual variava em função de diferentes experiências e predisposições inatas. Além de Edelman, muitos estudiosos atualmente têm posições semelhantes (cf. Barton, 1994; Bronfenbrenner and Ceci, 1994; Rosenfield, 1988; Schneider and Weinert, 1989).

A posição de Edelman com relação à compreensão atual do desenvolvimento biológico nos seres humanos foi assim resumida por Sylwester (1995):

O padrão genético básico de desenvolvimento para o nosso cérebro é, portanto, bastante simples e claro: (1) criar um excesso inicial de células e conexões entre áreas relacionadas — na verdade, ligar temporariamente tudo a tudo; (2) utilizar a emoção, a experiência e a aprendizagem para fortalecer as conexões úteis, e então eliminar as conexões não usadas e ineficientes, e (3) manter uma flexibilidade sináptica (comumente chamada de plasticidade) suficiente para permitir que as conexões da rede neural se modifiquem no decorrer da existência, na medida em que mudam as condições e surgem novos desafios que envolvem a solução de problemas. (p.126)

Uma característica adicional de nossa herança biológica afeta a natureza do desenvolvimento humano. Como foi criado por seleção natural, o cérebro humano não é projetado perfeitamente para atender a variadas demandas do ambiente (e.g. Dawkins, 1982), nem é um órgão para fins gerais, independente de domínio. Ao contrário, o cérebro é composto de diferentes áreas, ou módulos, e inteligências (este último termo será o utilizado de ora em diante) que controlam várias funções cognitivas e que são diferencialmente capazes de se beneficiar de experiências variadas. Quais são, portanto, as divisões estruturais e funcionais do cérebro que podem ser desenvolvidas? Uma classificação bem conhecida 
refere-se a sete inteligências distintas, descritas por Howard Gardner em 1983: as inteligências lingüística, musical, lógico-matemática, espacial, corporal-cinestética, interpessoal e intrapessoal. Recentemente, Gardner (1995, 1999) acrescentou uma oitava inteligência: a naturalista. Embora cada inteligência possa ser associada a uma ou mais áreas específicas do cérebro que são primordialmente responsáveis para mediar o conhecimento relevante, cada inteligência é também conectada de forma intricada com outras inteligências no ser humano normalmente integrado.

Portanto, seguindo Gardner, o desenvolvimento cognitivo pode ocorrer em oito inteligências distintas mas estreitamente ligadas. Além disso, o desenvolvimento reflete a crescente capacidade de cada um em domínios específicos de conhecimento, que são áreas de especialização criadas pelos seres humanos, mais do que uma habilidade cognitiva generalizada. Uma pessoa, por exemplo, poderá desenvolver a inteligência espacial tornandose competente em xadrez. Entretanto, o fato de alguém se tornar perito em xadrez não o torna um perito em reconhecimento de padrões visuais (Hirschfeld and Gelman, 1994). Além disso, não se deve presumir que o conteúdo de cada inteligência é igualmente fácil de ser absorvido ou transmitido (Cosmides and Tooby, 1994). Por último, as provas examinadas por Gardner sugerem que as inteligências se desenvolvem em ritmos diferentes e em idades diferentes, um ponto ao qual voltaremos abaixo.

Resumindo o argumento até aqui, o trabalho de Edelman e de outros sugere que as pessoas são equipadas, ao nascimento, para praticar qualquer esporte ou falar qualquer língua inventada pelos homens. Entretanto, o esporte que uma pessoa vai praticar ou a língua que vai falar depende dos ambientes físicos e culturais nos quais ela está imersa e da idade com a qual ela participa dessa atividade. O desenvolvimento humano não ocorre uniformemente ao longo da ampla faixa da capacidade humana mas, ao contrário, se realiza em ritmos diferentes dentro de um número de diferentes capacidades ou inteligências inatas.

\section{A heterogeneidade do desenvolvimento cultural}

Sobre o fundo comum da capacidade humana de desenvolver as predisposições biológicas encontra-se o fator cultural, que afeta a natureza e a direção específica do desenvolvimento cognitivo individual. São apresen- 
tadas a seguir três proposições que caracterizam o impacto da cultura no desenvolvimento.

\section{Proposição $\mathrm{n}^{0}$ 1: $\mathrm{O}$ desenvolvimento depende de significados mediados por signos.}

Durante os últimos cinqüenta anos, a maioria dos psicólogos e educadores ocidentais manteve o ponto de vista de que o desenvolvimento lidera a aprendizagem. Nessa perspectiva, a quantidade e os tipos de aprendizagem possíveis dependem de uma seqüência invariável de estágios que têm uma correspondência muito próxima com a idade cronológica. As teorias de Piaget e Kohlberg são exemplos dessa forma de entender o desenvolvimento humano. Entretanto, especialmente nos últimos 20 anos, tem havido freqüentes questionamentos dessas teorias de desenvolvimento por estágios (cf. Brainerd, 1978; Donaldson, 1979; Granott and Gardner, 1994; Phillips and Kelly, 1975; Schweder, Mahapatra, and Miller, 1990).

Um ponto de vista alternativo do desenvolvimento humano, baseado em diferentes linhas de pesquisa, é o de que a aprendizagem lidera o desenvolvimento. Nessa perspectiva, o "nível" de desenvolvimento de um indivíduo depende primordialmente de suas experiências de vida com os fenômenos em questão. À guisa de ilustração, os estudos baseados no paradigma iniciante-perito confirmam que as crianças que possuem um rico conhecimento específico de determinado domínio têm melhor desempenho que adultos que são iniciantes naquele mesmo domínio (e.g., Chi, 1978). Atualmente, existem muitas propostas sobre em que consiste a aprendizagem e sobre o que conta como evidência de aprendizagem. O ponto de vista cultural-psicológico aqui adotado é o de que a essência da aprendizagem é o significado que é derivado de uma atividade socialmente situada (Smith, 1995).

\section{Domínios do significado}

Apesar da crença inicial de Vygotsky de que o desenvolvimento ocorre dentro de sistemas funcionais particulares (cf. Scribner, 1985), o ponto de vista dominante no Ocidente tem defendido a uniformidade de 
crescimento em toda a ampla gama de capacidade cognitiva. Essa pressuposição transformou-se gradualmente nos últimos 25 anos em um reconhecimento dos muitos tipos de capacidade humana e da possibilidade de diferentes ciclos de crescimento para cada capacidade. Quais são as áreas em que o significado (leia-se "o desenvolvimento") ocorre a nível individual? Minha tese é a de que essas áreas correspondem às mesmas oito inteligências múltiplas descritas acima (Gardner, 1983), em que cada inteligência é ligada a sistemas culturais de signos, ou significados (Gardner utilizou o termo "sistemas simbólicos").

Com referência a um ponto apresentado anteriormente, Gardner propôs que diferentes seqüências de desenvolvimento operam com variada duração de tempo sobre cada inteligência. Em um capítulo menos conhecido de seu clássico livro de 1983, Gardner descreveu as seqüências relevantes de desenvolvimento como fluxos, ondas e canais. Ele definiu fluxo como "uma progressão que é única de cada sistema simbólico [de signos]" (p. 305), afirmando que "o desenvolvimento na forma de fluxo [é] a articulação de uma inteligência específica assim que a capacidade intelectual tenha se tornado suscetível de envolvimento em sistemas simbólicos [de signos] da cultura (ou suscetível de apropriação por tais sistemas)" (p. 306). Gardner definiu onda como um processo abrangente que começa tipicamente em um campo simbólico, ou de signos, mas que se espalha rapidamente, e às vezes de forma indevida, para outros domínios simbólicos (p. 307). Até o presente, foram descritas quatro ondas para tratar do mundo complexo do significado (Gardner, 1991; Gardner and Wolf, 1983). Por fim, Gardner descreveu canal como um meio de codificar as informações que evoluíram dentro de uma cultura e que são repassadas diretamente ao aprendiz. Mais recentemente, Granott e Gardner (1994) descreveram as características de um efeito de Inteligência Múltipla de segunda ordem que se desenvolve de forma diferenciada entre inteligências. A implicação do trabalho de Gardner e de seus colegas é não apenas que o significado se desenvolve dentro de várias inteligências humanas, mas que há várias e diferentes seqüências de desenvolvimento dentro de cada inteligência. Nesta perspectiva, não é mais sustentável a crença em apenas uma seqüência geral de desenvolvimento. 


\section{Signos}

Há alguns anos, Bruner (1964) defendeu o ponto de vista de que o desenvolvimento do funcionamento intelectual humano depende do "domínio de técnicas e não pode ser entendido sem referência a tal domínio. Essas técnicas não são, de forma geral, invenções de indivíduos que estão 'crescendo'; elas são, melhor dizendo, habilidades transmitidas pela cultura com vários graus de eficiência e de sucesso - sendo a língua o principal exemplo" (p.1). Mais adiante, no mesmo artigo, Bruner argumentou que "o crescimento depende do surgimento de duas formas de competência.... representação e integração. Tenho sugerido que podemos conceber o crescimento em ambos estes domínios como o surgimento de novas tecnologias para a liberação e a amplificação dos poderes intelectuais humanos" (1964, p. 13). Em minha opinião, as tecnologias a que Bruner se refere são o que os semioticistas chamam de signos (e.g., Buchler, 1955; Mertz and Parmentier, 1985; Smith, 1992) que operam através das inteligências delineadas acima.

A noção de signo adotada aqui é a de um processo triádico, irredutível e incessante enunciado por Charles Peirce em 1897, em um texto bem conhecido:

O signo, ou representamen, é algo que se representa alguma coisa a alguém, em alguma condição ou capacidade. Ele se endereça a alguém, ou seja, cria na mente daquela pessoa um signo equivalente, ou talvez um signo mais desenvolvido. O signo por ele criado eu o chamo de interpretante do primeiro signo. O signo representa alguma coisa, seu objeto. Está em lugar daquele objeto, não em todos os aspectos, mas em referência a algum tipo de idéia, que eu às vezes chamei de base do representamen. (Buchler, 1955, p. 99)

Sem tentar desenlaçar aqui as complexidades da formulação de Peirce, defendo a idéia de que o significado é a consequiência natural do processo de signos, e que são os signos que tornam possíveis os significados e o desenvolvimento humano. 


\section{Proposição $n^{0}$ 2: Os significados têm uma base cultural e são motivados por necessidades de competência}

Significado

A esta altura, o leitor atento deve ter percebido que o conceito de significado ainda precisa ser esclarecido. Para os objetivos deste trabalho, uma breve introdução será considerada adequada (uma discussão ampla do conceito pode ser encontrada em Ogden and Richards, 1946). Simplificando um pouco as coisas, há duas orientações na forma de se tratar o significado: uma abordagem formal, objetiva e abstrata, baseada na lógica, e uma abordagem informal, subjetiva e de base contextual, ligada às atividades humanas (Santambrogio and Violi, 1988). A abordagem formal é preferida por aqueles que, como Bertrand Russell, procuram desenvolver conjuntos abstratos de operações formais, a fim de estabelecer significados objetivos (ou seja, não pessoais) de sentenças ou proposições não contextualizadas. A abordagem informal é endossada por aqueles que, como Bartlett (1932), procuram considerar o significado no contexto de capacidades e funções humanas, as quais abrangem, entre outras coisas, as intenções comunicativas e as convenções culturais (cf. Bruner, 1990). A abordagem informal é a adotada neste trabalho.

O enfoque no significado, na psicologia cultural, é inteiramente coerente com os recentes progressos em teorias e pesquisas de diversos campos. Sinott (1993) resumiu de forma eloqüente esses avanços, da seguinte maneira: na física, a relatividade e a física quântica demonstraram que múltiplas realidades contraditórias podem ser todas verdadeiras, dependendo do ponto de vista; na biologia, a evolução cooperativa mostrou que as espécies não lutam pelo mesmo nicho, mas evoluem para se adequar a nichos inter-relacionados e não sobrepostos; na matemática, a teoria do caos dá suporte ao ponto de vista de que a desordem adaptiva acontece não por acaso e conduz a resultados imprevisíveis. Da mesma forma, no desenvolvimento humano, a analogia pertinente é de que os indivíduos escolhem e constróem suas próprias verdades e realidades.

A propósito, vale a pena observar que a idéia de significado não tem sido confinada à espécie humana. Barton (1994) descreveu um programa de pesquisa desenvolvido por Freeman, que investigou como os cheiros eram lembrados e representados no sistema de olfato dos ratos. Estudando os padrões espaciais nos eletroencefalogramas desses animais, Freeman descobriu que os padrões mutantes relativos ao cheiro de hortelã-pimenta sugeriam que "a representação neurológica de um cheiro não é fixa como uma fotografia. Pelo contrário, a estrutura da aprendizagem mais antiga se 
transforma no contexto da aprendizagem mais recente" (Barton, 1994, p. 9). Esta pesquisa mostrou que a fluidez da representação neurológica servia de evidência para a auto-organização das estruturas neorológicas, e indicava que essas estruturas espaço-temporais "refletem o significado [nosso grifo] do estímulo, e não apenas sua presença” (p. 9).

Para que serve a construção do significado? Uma importante pergunta que não é feita pelas teorias tradicionais de desenvolvimento por estágios é a seguinte: por que se desenvolver, ou, qual é o objetivo do desenvolvimento? Bruner (1986) considerou alguns elementos motivacionais à luz da perspectiva teórica de Piaget:

\footnotetext{
Ou, no caso de Piaget... não havia lugar para o uso e a intenção, apenas para uma análise dos produtos da mente, de forma abstrata. Portanto, não havia lugar para dilemas humanos, para condições de tragédia, para o conhecimento local encapsulado no preconceito. O próprio programa de Piaget, sua "epistemologia genética", era insuficientemente humano: traçar a história do matemática e da ciência no crescimento da mente da criança. Mas que luz isso lança sobre a história da sensibilidade, da "loucura", da alienação, ou das paixões?... Mais modestamente, o sistema não conseguiu capturar a particularidade do conhecimento do homem comum, o papel das negociações no estabelecimento dos significados. (p. 147)
}

A posição adotada aqui é de que a busca do significado e o crescimento do significado são motivados, no nível mais básico, pela necessidade dos seres humanos de sobreviver como entidades biológicas e culturais. As probabilidades de sobrevivência de um indivíduo aumentam se ele se tornar competente nas atividades do ambiente e da cultura ao seu redor (e.g. Smith, 1992; White, 1959). Assim é vista a crescente competência dos bebês mais novos que dão mostra de atos comunicativos extraordinários para permanecerem aquecidos e alimentados e para se tornarem partes importantes no meio sociocultural local (e.g. Bruner and Sherwood, 1981; Shotter, 1978). Evidentemente, diferentes culturas atribuem valores diferentes a certos signos e sistemas de signos (e.g. Gardner, 1983, 1991), os quais devem ser aprendidos, ou constituídos como significativos, por todos os membros daquela sociedade. Gardner (1991), por exemplo, sugeriu que "de forma geral, os ugandenses valorizam o envolvimento vagaroso e cuidadoso nas atividades, os mexicanos enfatizam a sensibilidade interpessoal, e os chineses dão grande importância ao domínio de grandes volumes de informação factual" (p. 82). Mais especificamente, Granott e Gardner (1994) fizeram uma resenha das pesquisas que demonstravam que: (a) crianças mexicanas provindas de famílias que 
trabalhavam com cerâmica tinham um desempenho significativamente melhor, nas tarefas de conservação de Piaget, do que seus pares de idade, escolaridade e classe socioeconômica semelhantes, (b) crianças australianas aborígenes demonstraram melhor desempenho em tarefas espaciais relacionadas com a exploração do terreno do que em tarefas lógico-matemáticas, ocorrendo o contrário com as crianças européias, e (c) muitas crianças japonesas que aprenderam a tocar violino utilizando o método Suzuki eram capazes de tocar um concerto de Vivaldi aos seis anos de idade.

\section{Proposição $n^{0}$ 3: Os significados individuais são negociados continuamente na atividade cultural}

Vários elementos ligados a esta proposição serão brevemente resumidos, independentemente de sua ordem de importância. Em primeiro lugar, os significados surgem de atividades, e em atividades com outros membros da cultura (e.g., Lave and Wenger, 1991). Essas atividades são culturalmente valorizadas e normalmente ligadas à sobrevivência da cultura. Em segundo lugar, ao nível individual, o crescimento do significado dura a vida toda. E como essas construções continuam durante a vida, as capacidades do início da infância não são particularmente capazes de fazer prever os talentos da idade adulta nem são irreversíveis (cf. Sinnott, 1993). Em terceiro lugar, dentro de um contexto cultural específico, o desenvolvimento é caracterizado por uma diversificação crescente entre os indivíduos. Grande parte desta diversificação é criada através de decisões participativas dos próprios indivíduos à medida que negociam seus lugares dentro de seu próprio meio (Bronfenbrenner and Ceci, 1994; Granott and Gardner, 1994; Hartup, 1989; Kessen, 1983; Scarr, 1992).

Pesquisas sobre ambientes não compartilhados. Scarr (1992) tem apresentado insights muito úteis em sua ampla análise de pares de irmãos geneticamente idênticos criados juntos ou separadamente, e sobre pares de irmãos adotivos criados juntos. A idéia mais comum é a de que um conjunto de características familiares que constituem um ambiente social compartilhado tem grande impacto nas capacidades em desenvolvimento da criança. Entretanto, em sua pesquisa, Scarr descobriu que as diferenças entre os pais, no que se refere a estilo de educação, renda e classe social, tinham efeitos menores nas diferenças mensuráveis entre as crianças no que se refere a inteligência, interesses e personalidade. Ela concluiu que os dados não confirmam a típica teoria do desenvolvimento, já que a maioria da variação ambiental resultava de fatores ambientais não compartilhados. 
Scarr (1992) concluiu que esses últimos fatores, ou seja, as influências que são diferentes para cada membro da família, resultavam das construções únicas do indivíduo em interações com outras pessoas, das oportunidades ignoradas ou aproveitadas, e dos significados atribuídos aos eventos.

Em sua meticulosa análise, Plomin, Chipuer e Neiderhiser (1994) concluíram que, quando comparado com os dotes genéticos ou com os ambientes sociais compartilhados, o ambiente não compartilhado (ou seja, o conjunto de influências ambientais experimentadas de forma diferente pelos membros de cada família) é a principal fonte de influência ambiental para importantes campos do comportamento, entre eles a psicopatologia e as habilidades cognitivas. Por exemplo, embora se acreditasse anteriormente que os escores de QI eram influenciados primordialmente por fatores ambientais compartilhados, dados recentes sugerem que isto é verdade apenas na infância e que, já na adolescência, os efeitos ambientais compartilhados são insignificantes.

Outro estudo (Dunn and McGuire, 1994) provou que crianças de uma mesma família obtiveram efeitos diferenciais marcantes devido a: (a) relacionamento com os pais, (b) o próprio relacionamento entre os irmãos, (c) relacionamento com amigos e professores fora da família, e (d) incidentes casuais, como doença ou morte de outros membros da família. Os resultados da pesquisa de Dunn e McGuire indicam que as crianças são construtoras ativas de seu próprio mundo, podendo influenciar as interações dentro de seus ambientes culturais e por elas ser influenciadas. A maior implicação destes resultados é que o desenvolvimento das capacidades das crianças se baseia essencialmente nos significados que cada criança constrói em seu próprio mundo.

Resumindo esta seção, pode-se afirmar que hoje a maioria dos estudiosos de psicologia cultural aceitam que o crescimento dos significados (e portanto o desenvolvimento) é multidirecional, sem um curso específico de maturação (cf., Bronfenbrenner and Ceci, 1994; Kessen, 1983). O crescimento individual é definido de acordo com as metas da comunidade local e é moldado tanto pelas forças biológicas como pelas aspirações culturais (e.g., Rogoff, 1990). O desenvolvimento ocorre dentro de um número de inteligências integradas mas potencialmente separáveis. 


\section{Implicações para uma educação multicultural}

São apresentadas a seguir cinco implicações para o ensino, a partir da posição de que desenvolvimento é a construção de significados, como resultado da utilização de signos de determinada cultura. Algumas dessas implicações são semelhantes àquelas já propostas por outras tradições teóricas e de pesquisa. Entretanto, são apresentadas aqui por motivos diferentes e admitem uma gama mais diversificada de tipos de experiências como formas legítimas de aprendizagem. Por exemplo, as formas de estágio, de aprendizagem substitutiva, e o prestar atenção à sabedoria dos mais velhos são consideradas formas tão valiosas de aprendizagem como os exercícios escolares formais. E como a palavra "aprendizagem" é utilizada abaixo no sentido de aprendizagem significativa, os termos "aprendizagem", "significado" e "construção do significado" serão utilizados como sinônimos.

\section{Reconhecer que o significado é cultural e contextual}

A visão de desenvolvimento aqui proposta reconhece que toda aprendizagem, ou significado, é adquirida em contextos específicos. Os contextos relevantes são de natureza tanto cultural quanto situacional e envolvem fatores tais como "quem", "quando", "onde", "o quê", e "por quê". Os significados derivados de situações particulares têm como resultado produtos mentais (alguns diriam estruturas cognitivas) que podem ou não se refletir em medidas simplistas de desempenho. Trata-se de uma consideração geral, mas que é de particular importância quando se consideram os testes de inteligência, por exemplo (e.g., McClelland, 1973).

Mesmo dentro de uma comunidade cultural específica, o contexto da aprendizagem é crítico. A base contextual da aprendizagem tem sido bem justificada por Brown, Collins e Duguid (1989). Além disso, Rogoff (1984) argumentou que os fatores contextuais desempenham um papel crítico nas atividades cognitivas e que, mesmo em um nível contextual mais baixo, "as habilidades não são empregadas de forma independente do contexto no qual se situa o problema" (Rogoff, 1984, p. 6). A tendência a sobrevalorizar as habilidades intelectuais fora de contexto tem resultado em teorias enganosas e em juízos equivocados sobre o leque de capacidades cognitivas das crianças (Donaldson, 1979). Portanto, os responsáveis pela instrução devem estar bem conscientes da especificidade 
contextual e da limitada capacidade de generalização de grande parte dos conteúdos das suas lições.

\section{Reconhecer o papel central dos esforços de grupo na construção de significados}

O reconhecimento de que o significado é contextual é apenas uma parte, e talvez uma parte pequena, do quadro completo. A construção do significado é também dirigida por projetos culturais mais amplos, que podem ser de natureza explícita ou implícita. Apesar da ênfase dada ao individualismo pelo sistema escolar formal do Ocidente, as pessoas não se constituem em ilhas. Os alunos pertencem a muitos outros ambientes além da escola, são construtores significativos de seus próprios ambientes (Scarr, 1992), e são grandemente influenciados por fatores constantes, como os pais (Clarke and Clarke, 1989). Na escola, os professores devem reconhecer o valor da aprendizagem cooperativa entre os pares, com o reconhecimento de que o conhecimento é social, negociado e distribuído.

Roschelle e Clancey (1992) apresentaram uma perspectiva útil para o ensino e a aprendizagem da física que merece ser examinado em outros contextos. Estes autores descreveram uma abordagem construtivista do conhecimento que enfoca como as representações são criadas, recebem significado e são utilizadas em um ambiente social específico. Em vez de receber formalismos abstratos em física, os alunos trabalharam juntos para compreender fenômenos de que eram parte. Além disso, Roschelle e Clancey recomendam que os alunos iniciantes de física devam primeiramente aprender a negociar o significado, utilizando objetos concretos manipuláveis, já que eles não têm um vocabulário técnico que lhes permita falar abstratamente do tópico em questão. Outros pesquisadores (e.g., Farr, 1981) têm assinalado que a mente humana emerge a partir da interação com outros e que a consciência de si mesmo se baseia na experiência social.

As influências sociais operam também em outras dimensões. Por exemplo, quando se comparam com outros, os aprendizes estão bem conscientes de suas forças e de suas fraquezas. Por isso, os educadores devem se lembrar que todas as forças e fraquezas são culturalmente relativas. Por si só, o indivíduo não é nem capaz nem incapaz (McDermott and Varenne, 1995) e os talentos humanos escolhidos para serem rotulados dessa forma são especificados culturalmente. A interação social também envolve as emoções, elemento central de grande parte da aprendizagem e da memória. Finalmente, o enfoque nos grupos sociais chama a atenção para 
o importante papel da memória externa e dos recursos na vida diária e do mundo do trabalho. Existe aqui um empréstimo e um compartilhamento do conhecimento de outros para trazer à existência produtos que nenhuma pessoa poderia criar por si só. Embora o sistema escolar tradicional do Ocidente veja a aprendizagem como essencialmente privada e individual, o envolvimento de grupos sociais nos faz lembrar que a construção do significado se baseia em perspectivas públicas e coletivas em que a cooperação e o dividir com os outros são elementos centrais do desenvolvimento humano.

\section{Proporcionar uma variedade de tarefas para uma variedade de campos de conhecimento}

Como foi exposto acima, os seres humanos possuem uma série de sistemas de habilidades, quase todos em constante interação com o ambiente sociocultural. Embora a escola formal enfatize o saber ler, escrever e calcular, os alunos só se tornarão integralmente competentes na sociedade mais ampla que a escola se eles se tornarem capazes nos sistemas de signos de inteligências múltiplas. Nesta perspectiva, Gardner (1983) proclamou que "a introdução e o domínio dos sistemas simbólicos são ... uma grande tarefa da infância e podem até ser considerados como a principal missão dos sistemas educacionais modernos" (p. 302).

A forma aqui esboçada de conceber os sistemas de habilidade humana e os sistemas de signos que lhe são associados oferece a possibilidade de representar o mesmo significado dentro de vários e diferentes sistemas de signos. Por exemplo, os estudantes que têm pouca habilidade lingüística podem ser incentivados a atuarem primeiro na área musical, espacial ou cinestésico-corporal, para só depois tentar representar o significado correspondente através da linguagem.

O processo de passar de um sistema de signos para outro tem sido chamado de "transmediação" (Siegel, 1995) e deve constituir um importante conjunto de objetivos para as tarefas de sala de aula. Este procedimento foi utilizado por Griss (1994), que relatou o uso do movimento criativo como instrumento no ensino de tópicos em áreas tão diversas quanto a ciência, a matemática, a literatura e os estudos sociais. Alternativamente, os alunos podem ser autorizados a apresentar seu trabalho em um formato não típico. Lee (1994), por exemplo, permitiu que uma aluna de pós-graduação utilizasse a dança para abordar os conceitos de consulta e colaboração. Sobre isso, Lee escreveu: "Mas eu não queria saber quão 
'inteligente' Janet era de acordo com um padrão pré-determinado; eu queria saber como ela era inteligente" (p. 83).

Decorre da discussão anterior que os professores devem utilizar sua instrução para envolver os alunos em alguma forma de uso autêntico, ao invés de focalizar apenas afirmações teóricas abstratas e descontextualizadas. Mais tecnicamente, isto significa incorporar os aspectos pragmáticos do material a ser aprendido, além de seus traços sintáticos e semânticos (Smith, 1992). Um professor de ciências, por exemplo, poderá atribuir como tarefa (ou, melhor ainda, fazer com que os alunos criem) um projeto que represente o que os cientistas fazem na prática, ao invés de enfocar problemas livrescos com uma única resposta correta. Uma ênfase desse tipo parece particularmente apropriada para aqueles indivíduos que nunca se sairão bem nas tarefas acadêmicas tradicionais e que não pretendem trilhar uma carreira profissional ou curso superior em que apenas aqueles atributos são valorizados. Tanto Scarr (1992) quanto Roschelle e Clancey (1992) endossariam a idéia de muitos tipos de experiências diversificadas na escola. Esta postura não deve excluir tarefas desenvolvidas a partir de concepções neo-piagetianas, ou outras de desenvolvimento (cf. McKeough, 1991), desde que alguns alunos possam delas tirar proveito.

\section{Ensinar com vistas à compreensão}

Uma grande implicação para o ensino, que decorre do processo de desenvolvimento dinâmico e contínuo aqui defendido, é a de ensinar com vistas à compreensão. Não se trata de uma recomendação nova. Entretanto, o ponto de vista ainda predominante afirma que a aprendizagem consiste de habilidades hierárquicas que devem ser agrupadas em pacotes de fácil consumo para os alunos que estejam passando por um ou outro estágio de desenvolvimento. $\mathrm{O}$ ensino com vistas à compreensão reconhece a natureza multidirecional da aprendizagem e a construção do significado que deve ocorrer para todos os aprendizes, inclusive o professor. Entretanto, a notícia de que o professor americano médio lê apenas um livro por ano (Gardner, 1991) não é muito alentadora para os professores que procuram ensinar com vistas à compreensão.

Através de seus escritos e de sua ligação com o Projeto Teaching for Understanding (Ensino com Vistas à Compreensão) de Harvard, Gardner (1991) tem atuado no sentido de conclamar uma abordagem educacional que defenda o desenvolvimento da compreensão nas crianças. Várias premissas decorrem desta perspectiva de aprendizagem: (a) recomenda-se que haja perspectivas múltiplas a respeito de cada tarefa; (b) são utilizadas 
tanto tarefas individuais como de grupo; (c) diferentes papéis são assumidos em sala de aula tanto pelos alunos como pelo professor, devendo este último servir de modelo de uma aprendizagem contínua; (d) a matéria escolar tem a ver diretamente com as vidas dos alunos e os engaja ativamente em contextos significativos; (e) é enfatizada uma auto-avaliação contínua do processo, em vez de uma avaliação apenas do produto, e (f) as idéias dos alunos são levadas a sério e atendidas de forma honesta.

O Projeto de Harvard, mencionado acima, propõe um trabalho de sala de aula baseado em quatro pontos (cf., Perkins and Blythe, 1994): (a) tópicos gerativos, em que o núcleo do currículo seja formado por tópicos selecionados que sejam acessíveis para os alunos, de ligação fácil com outros tópicos diversos, e centrais para a disciplina em questão; (b) compreensão de metas, em que os objetivos sejam especificados na forma de compreensão de tópicos específicos; (c) compreensão de desempenhos, em que os alunos se engajem em uma variedade de desempenhos (em vez de apenas tarefas), e (d) avaliação contínua.

Estes princípios e programas relevantes estão começando a aparecer em uma variedade de escolas. Torna-se claro que as salas de aula relevantes funcionam de forma diferente quando estas premissas são adotadas. Segundo Wiske (1994), "o ensino com vistas à compreensão exige uma negociação aberta e explícita sobre o que é o conhecimento, como pode ser desenvolvido e defendido, conhecimento de quem que conta, e como pode ser avaliado" (p. 19). Nelson (1994) demonstrou que até crianças do primeiro ano do ensino fundamental são capazes de criar seu próprio currículo, juntamente com as atividades pertinentes que sejam melhor adequadas às suas capacidades atuais. Haas e LoPresto (1994) relataram um projeto em que os alunos explicam as soluções de problemas matemáticos complexos diante de um grupo de juízes. Todas estas iniciativas favorecem o desenvolvimento da competência dos alunos, em signos culturais diversos, proporcionando-lhes tarefas autênticas que eles tornam significativas.

Há várias outras implicações para a educação multicultural que decorrem de um enfoque sobre a construção do significado e da compreensão. Em primeiro lugar, é importante evitar as limitações da "valeta disciplinar". Embora os professores tendam a promover uma visão culturalmente aceita de suas disciplinas, especialmente nos níveis de ensino médio e superior (cf., Roschelle and Clancey, 1992), os alunos devem ser incentivados a compreender as várias interrelações entre as disciplinas, e a utilizar essas ligações para aprimorar sua própria construção de significados. 
Em segundo lugar, os professores devem se dar conta de que a aprendizagem não é baseada em hierarquias. Ao contrário, a aprendizagem é suficientemente complexa para ser multidirecional e multifacetada em todas as tarefas, excetuadas apenas as mais simples. Como o que ocorre em nosso cérebro, tudo está ligado a tudo. Não há motivo para presumir que a aprendizagem de um fato é condição para que outro fato possa ser aprendido (e.g., Phillips and Kelly, 1975) ou que há uma estrada única para o significado e para o desenvolvimento.

Em terceiro lugar, o currículo não deve ser um conjunto rígido de conhecimentos fixos. Ao contrário, deve-se reconhecer o currículo como algo que está em evolução constante dentro da cultura ou, até mesmo, como "cultura em construção". Além disso, o currículo é constantemente negociado por alunos e professores nas salas de aula. Sabe-se há algum tempo que um currículo aprovado em uma instância governamental toma várias formas assim que chega às salas de aula. O "mesmo" currículo pode ser representado como o currículo aprovado, o currículo ensinado, e o currículo "que pegou".

Por fim, professores e alunos devem aceitar o fato de que há múltiplas perspectivas sobre a verdade e a realidade. Embora as culturas tenham a tendência de desenvolver e dar preferência a suas próprias perspectivas sobre o mesmo conjunto de fenômenos, é ainda verdade que um prisma tem muitas facetas. Os professores devem ser incentivados a estar receptivos a uma variedade de perspectivas a fim de fomentar a construção de múltiplos significados em suas salas de aula. Por sua vez, este processo deve dar suporte ao máximo desenvolvimento das capacidades cognitivas humanas.

\section{Reconhecer os interesses e os pontos fortes individuais}

Conscientes das premissas de que os indivíduos constróem seus próprios ambientes (Scarr, 1992), de que os indivíduos possuem uma série de pontos fortes que lhes são únicos (Gardner, 1983), e que o desenvolvimento ocorre em contextos socioculturais (e.g., Brown et al., 1989; Bruner, 1990), os professores devem reconhecer os talentos especiais de seus alunos e utilizá-los da melhor forma possível. Nesta linha, os alunos devem ser incentivados a construir significados dentro de seus sistemas de signos preferidos ou nos quais eles tenham mais potencial. Entretanto, deve-se acentuar que o que está sendo defendido aqui não é uma reencarnação do movimento de instrução individualizada, que foi popular durante 
os anos 70. A instrução individualizada não tinha entre suas premissas a construção de significado por parte dos alunos.

Embora haja uma associação entre faixa etária e interesses específicos (cf., Gardner, 1983), dentro de uma mesma faixa os indivíduos variam muito em termos de suas experiências anteriores e de sua prontidão para aprender de pacotes curriculares fixos. A expressão muito citada de que "deve-se começar do ponto em que se encontra a criança" continua a ter validade. Testes padronizados de QI devem ser relegados, dando-se preferência à criação de pastas de trabalhos produzidos e outras formas de avaliação autêntica. Os professores devem respeitar os alunos como pensadores e como aprendizes, mesmo quando esses tenham dificuldades com as tarefas escolares (Donaldson, 1979).

À guisa de conclusão, os professores que prestarem atenção às sugestões apresentadas estarão contribuindo para que seus alunos se tornem competentes em um conjunto variado de signos de suas culturas ambientes. As crianças de culturas minoritárias deverão ser capazes de participar plenamente dos trabalhos em tais salas de aula e de desenvolver seu potencial biológico e cognitivo como todas as demais.

\section{RESUMO}

O principal objetivo deste artigo é defender a tese de que: (a) todos os seres humanos são dotados do mesmo potencial biológico para sobreviver em meios físicos e culturais diversos, e (b) o desenvolvimento humano depende mais de significados criados a partir de experiências pessoais em contextos culturais do que de traços biológicos ligados à idade. Como segundo objetivo deste trabalho, e com base nos dois argumentos acima, são apresentadas cinco implicações gerais para a educação em contextos multiculturais.

\section{REFERÊNCIAS BIBLIOGRÁFICAS}

BARTLETT, F.C. Remembering: A Study in Experimental and Social Psychology. Cambridge: Cambridge University Press, 1932. 
BARTON, S. Chaos, self-organization, and psychology. American Psychologist, n. 49, p. 514, 1994.

BRAINERD, C. J. The stage question in cognitive-developmental theory. Behavioral and Brain Sciences, n. 2, p. 173-213, 1978.

BRONFENBRENNER, U. and CECI, S.J. Nature-nurture reconceptualized in developmental perspective: A bioecological model. Psychological Review, n. 101, p. 568-586, 1994.

BROWN, J. S.; COLLINS, A.; DUGUID, P. Situated cognition and the culture of learning. Educational Researcher, v. 18, n. 1, p. 32-42, 1989.

BRUNER, J. The course of cognitive growth. American Psychologist, n.19, p. 1$15,1964$.

Actual minds, possible worlds. Cambridge. MA: Harvard University Press, 1986.

. Acts of meaning. Cambridge, MA: Harvard University Press, 1990. and SHERWOOD, V. Thought, language and interaction in infancy. In: J. P. FORGAS (Ed.), Social cognition: Perspectives on everyday understanding. London: Academic Press, p. 27-52, 1981.

BUCHLER, J. Philosophical writings of Pierce. New York: Dover, 1955.

CHI, M. T. H. Knowledge structures and memory development, 1978.

CLARKE, A. M., and CLARKE, A. D. B. The later cognitive effects of early intervention. Intelligence, n. 13, p. 289-197, 1989.

COLE, M. Cognitive development and formal schooling: the evidence from crosscultural research. In: L. C. MOLL (Ed.), Vygotsky and education: Instructional implications and applications of sociohistorical psychology. Cambridge: Cambridge University Press. p. 89-110, 1990.

COSMIDES, L. and TOOBY, J. Origins of domain specificity: the evolution of functional organization In: L. A. HIRSCHFELD and S. A. GELMAN (Eds.), Mapping the mind: Domain specificity in cognition and culture. Cambridge: Cambridge University Press, p. 85-116, 1994.

DAWKINS, R. The extended phenotype. San Francisco: W. H. Freeman, 1982.

DONALDSON, M. The mismatch between school and children's minds. Human Nature. n. 2, p. 60-67, 1979.

DUNN, J. and McGUIRE, S. Young children's nonshared experiences: A summary of studies in Cambridge and Colorado. In: E. M. HETHERINGTON, D.; REISS, and R. PLOMIN (Eds.), Separate social worlds of siblings: the impact of nonshared environment on development. Hillsdale, N J: Erlbaum, p. 111128, 1994.

EDELMAN, G. M. Neutral Darwinism: The theory of neuronal group selection. New York: Basic Books, 1987.

Bright air, brilliant fire: On the matter of the mind. New York: Basic Books, 1992.

FARR, R. The social origins of the human mind: a Historical note: In: J. P. FORGAS (Ed.), Social cognition: Perspectives on everyday understanding. London: Academic Press, p. 247-258, 1981. 
GARDNER, H. Frames of Mind: The theory of multiple intelligences. New York: Basic, 1983

. The unschooled mind: How children think and how schools should teach.

New York: BasicBooks, 1991.

Reflections on multiple intelligences: Myths and messages. Phi Delta Kappan, n. 77, p. 200-209, 1995.

. Are there additional intelligences? The case for naturalists, spiritual and

existential intelligences. In: J. KANE (Ed.), Education, information, and transformation: Essays on learning and thinking. Upper Saddle River, N J: PrenticeHall, p. 111-132, 1999.

GARDNER, H. and WOLF, D. Waves and streams of symbolization: notes on the development of symbolic capacities in young children. In: D. ROGERS and J. A. SLOBODA (Eds.), The acquisition of symbolic skills. New York: Plenum Press, p. 19-42, 1983.

GRANOTT, N. and GARDNER, H. When minds meet: interactions, coincidence, and development in domains of ability. In: R. J. STENBERG e R. K. WAGNER (Eds.), Mind in Context: Interactions perspectives on human intelligence. Cambridge: Cambridge University Press, p. 171-201, 1994.

GRISS, S.Creative movement: a language for learning. Educational Leadership, v. 51, n. 5, p. 78-80, 1994.

HAAS, N. M. and LoPRESTO, S. Panel assessments. Unlocking math exams. Educational Leadership, v. 51, n. 5, p. 69-70, 1994.

HARTUP, W. W. Social relationships and their developmental significance. American Psychologist, n. 44, p. 120-126, 1989.

HIRSCHFELD, L. A. and GELMAN, S. A. Toward a topography of mind: An introduction to domain specificity. In: L. A. HIRSCHFELD and S. A. GELMAN (Eds.), Mapping the mind: Domain specificity in cognition and culture. Cambridge: Cambridge University Press, p. 3-35, 1994.

KESSEN, W. The child and other cultural inventions. In: F. S. KESSEL and A. W. SIEGEL (Eds.), The child and other cultural inventions. New York: Praeger, p. 26-39, 1983.

LAVE, J., and WENGER, E. Situated learning: Legitimate peripheral participation. Cambridge University Press, 1991.

LEE, P. A. To dance one's understanding. Educational Leadership, v. 51, n. 5, p. 81-83, 1994.

McCLELLAND, D. C. Testing for competence rather than for "intelligence." American Psychologist, n. 28, p. 1-14, 1973.

McDERMOTT, R. and VARENNE, H. Culture as disability. Anthropology and Education Quarterly, n. 26, p. 324-348, 1985.

McKEOUGH, A. Neo-piagetian theory goes to school: program development and program delivery. Exceptionality Education Canada, v. 1, n. 4, p. 1-17, 1991.

MERTZ, E. and PARMENTIER, R. J. (Eds.). Semiotic mediation: Sociocultural and psychological perspectives. Orlando, FL: Academic Press, 1985. 
NELSON, J. R. Can children design curriculum? Educational Leadership, v. 51 n. 5, p. 69-70, 1994.

OGDEN, C. K. and RICHARDS, I. A. The meaning of meaning, 8. ed. New York: Hartcourt, Brace and World, 1946.

PERKINS, D. and BLYTHE, T. Putting understanding up front. Educational Leadership, v. 51, n. 5, p. 4-7, 1994.

PHILLIPS, D. C., and KELLY, M. E. (1975). Hierarchical theories of development in education and psychology. Harvard Educational Review, n. 45, p. 351-375, 1975.

PLOMIN, R.; CHIPUER, H. M. and NEIDERHISER, J. M. Behavioral genetic evidence for the importance of nonshared environment. In: E. M. HET, 1994.

ERINGTON, D.; REISS and R. PLOMIN (Eds.), Separate social worlds of siblings: the impact of nonshared environment on development. Hillsdale, NJ: Erlbaum, p. 1-31.

ROGOFF, B. Introduction: thinking and learning in social context. Cambridge, MA: Harvard University Press, p. 1-8, 1984.

. Apprenticeship in thinking: cognitive development in social context. New York: Oxford University Press, 1990.

ROSCHELLE, J.; CLANCEY, W. J. Learning as social and neural. Educational Psychologist, n. 27, p. 435-453, 1992.

ROSENFIELD, I. The invention of memory: a new view of the brain. New York: Basic, 1988.

SACKS, O. Making up the mind. New York Review of Books, p. 42-49, 1993, Apr. 8.

SANTAMBROGIO, M. and VIOLI, P. Introduction. In: ECO, U.; SANTAMBROGIO, M. and VIOLI P. (Eds.) Meaning and mental representations. Bloomington: Indiana University Press, p. 3-22, 1988.

SCARR, S. Developmental theories for the 1990s: development and individual differences. Child Development, n. 63, p. 1-19, 1992.

SCHNEIDER, W. and WEINERT, F. E. Universal trends and individual differences in memory development. In: RIBAUPIERRE, A. de (Ed.). Transition mechanisms in child development: the longitudinal perspective. Cambridge: Cambridge University Press, p. 68-106, 1989.

SCRIBNER, S. Vigotsky's uses of history. In: WERTSCH, J. V. (Ed.), Culture, communication, and cognition: Vygotskian perspectives. Cambridge: Cambridge University Press, p. 119-145, 1985.

SHOTTER, J. The cultural context of communication studies: theoretical and methodological issues. In: LOCK, A. (Ed.). Action, gesture and symbol: the emergence of language. London: Academic Press, p. 43-78, 1978.

SHWEDER, R. A. MAHAPATRA, M. and MILLER, J. G. Culture and moral development. In: STIGLER, J. W.; SHWEDER, R. A. and G. HERDT (Eds.). Cultural psychology: essays on comparative human development. Cambridge: Cambridge University Press, p. 130-204, 1990. 
SIEGEL, M. More than words: the generative power of transmediation for learning. Canadian Journal of Education, n. 20, p. 455-475, 1995.

SIEGLER (Ed.), Children's thinking: What develops? Hillsdale, N J: Erlbaum.p. 73-96.

SINNOTT, J. D. Yes, it's worth the trouble! Unique contributions form everyday cognitive studies. In: PUCKETT, J. M. and REESE, H. W. (Eds.). Mechanisms of everyday cognition. Hillsdale, NJ: Erlbaum, p. 73-94, 1993.

SMITH, H. A. Semiotics ad modern schooling. In: BALAT, M. and DELEDALLE-RHODES, J. (Eds.). Signs of humanity. Berlin: Mouton de Gruyter, v. 1, p. 121-126, 1992.

SMITH, H. A. Cultural psychology and semiotics: confronting meaning in educational practice. Canadian Journal of Education, n. 20, p. 407-414, 1995.

SYLWESTER, R. A celebration of neurons: an educator's guide to the human brain. Alexandria, VA: Association for Supervision and Curriculum Development, 1995.

VYGOTSKY, L. Mind in society: the development of higher psychological processes. Cambridge, MA: Harvard University Press, 1978.

WHITE, R. W. Motivation reconsidered: the concept of competence. Psychological Review, n. 66, p. 297-333, 1959.

WISKE, M. S. How teaching for understanding changes the rules in the classroom. Educational Leadership, v. 51, n. 5, p. 19-21, 1994. 